\title{
SELETIVIDADE DE HERBICIDAS, APLICADOS EM PÓS-EMERGÊNCIA, ÀS PLANTAS DE ALFAFA ${ }^{1}$
}

\author{
GLÁUCIA DE MELLO ${ }^{2}$, RICARDO A. REIS ${ }^{3}$, JULIO C. DURIGAN ${ }^{4}$ e LINO R. FERREIRA ${ }^{5}$
}

\section{RESUMO}

Foram conduzidos doi experimentos na UNESP/Jaboticabal, em casa-de-vegetação, com o objetivo de avaliar-se a toxicidade de diferentes herbicidas, aplicados em pós-emergênica, às plantas de alfafa. No primeiro, utilizou-se de um delineamento experimental com parcelas inteiramente casualizadas para avaliar-se os efeitos fitotóxicos dos seguintes herbicidas: MSMA, clethodim + óleo mineral, lactofen, fluazifop-pbutil, fomesafen + óleo mineral haloxyfop-methyl + óleo mineral, fenoxaprop-ethyl, chlorimuronethyl, halosulfuron + óleo mineral, nicosulfuron, acifluorfen, imazethapyr, bentazon + óleo mineral e cyanazine + simazine, todos em só dose. Foram feitas avaliações da fitotoxicidade por meio de notas, atribuídas visualmente, em função dos sintomas constatados nas plantas. A altura das plantas e o peso da matéria seca da parte área foram avaliadas no período de desenvolvimento inicial e após a primeira rebrota. No segundo experimento foi seguido mesmo esquema de instalação e condução do anterior, após escolherse os herbicidas e doses, sendo eles, o MSMA, chlorimuron-ethyl, imazethapyr, bentazon + óleo mineral, clethodim, clethodim + óleo mineral e bentazon + MSMA. Os herbicidas mais seletivos às plantas de alfafa foram haloxyfop-methyl, fluazifop-p-butil, fenoxaprop-ethyl, MSMA, imazethapyr, bentazon e clethodim isolado e adicionado de óleo mineral)

Palavras chave: Medicago sativa, casade-vegetação, controle químico.

\section{ABSTRACT \\ Selectivity of applied port-emergence herbicides in the alfafa plants}

Two experiments were carried out in a greenhouse, belonging to the Departamento de Defesa Fitissanitária da Faculdade de Ciências Agrárias e Veterinárias de Jaboticabal/UNESP, with the purpose of evaluating the selectivity of different used post-emergence herbicides on alfafa plants. The experimental design was completely randomized to evaluate the phytotoxical effects of following herbicides: MSMA, clethodim plus mineral oil, lactofen, fluazifop-p-butil, fomesafen plus mineral oil, haloxyfop-methyl plus mineral oil, chlorimuron-ethyl, halosulfuron plus mineral oil, nicosulfuron, acifluorfen, imazethapyr, bentazon plus mineral oil and cyanazine plus mineral oil. The herbicides visual phytotoxical effects, plant height, and shoot production of dry matter, on the period of initial development and after the first regrowth of these plants were evaluated. $\mathrm{N}$ the second experiment, the same scheme for installing and carrying out was used, using different doses of MSMA, chlorimuronethyl, imazethapyr, bentazon, bentazon plus mineral oil, clethodin, clethodim plus mineral oil and bentazon plus MSMA. The most selective herbicides for alfafa were haloxyfop-methyl, fluazifop-p-butil, fenoxaprop-ethyl, MSMA,

\footnotetext{
${ }^{1}$ Recebido para publicação em 27/06/97 e na forma revisada em 05/04/2000.

${ }^{2}$ Eng $^{\mathrm{a}} \mathrm{Agr}^{\mathrm{a}}$, Faz. Santo Antonio, CEP: 14795-000, Colômbia/SP.

${ }^{3}$ Zoot., Prof ${ }^{\circ}$ Dr., FCAV/UNESP, CEP: 14870-000, Jaboticabal/SP.

${ }^{4}$ Eng $^{\mathrm{o}}$ Agr $^{\mathrm{o}}$, Prof ${ }^{\mathrm{o}}$ Dr., FCAV/UNESP, CEP: 14870-000, Jaboticabal/SP.

${ }^{5}$ Eng $^{\mathrm{o}}$ Agr $^{\mathrm{o}}$, Prof ${ }^{\mathrm{o}}$ Dr.,UFV, CEP: $36571-000$, Viçosa/MG.
} 
imazethapyr, bentazon and clethodim. (isolated and addicionated of mineral oil).

\section{INTRODUÇÃO}

A alafafa (Medicago sativa L.) é uma leguminosa perene, de clima temperado, com elevado valor nutritivo e grande capacidade de produção de forragem durante o ano. Dentre os fatores que limitam a produção desta leguminosa, destacam-se os relacionado à fertilidade do solo, à disponibilidade de água e à interferência exercida pelas plantas daninhas, acarretando em alto custos de produção (Honda \& Honda, 1990). Segundo Dawson \& Rincker (1982), dispende-se aproximadamente 930 horas/ha para manter a cultura da alfafa livre de plantas daninhas com capinas manuais.

O controle das plantas daninhas, além de eficaz, presta-se bem às áreas extensas, não proporciona danos ao sistema radicular das plantas, pode ser utilizado em épocas de grande precipitação pluviométrica e tem custo competitivo (Durigan, 1983). No entanto, são necessárias as informações relativas à seletividade de tais produtos químicos às plantas da cultura.

O uso de herbicidas é uma prática rotineira nos países de clima temperado, porém, no Brasil, ainda não existem recomendações seguras destes produtos químicos para a cultura da alfafa, sobretudo daqueles com ação sobre as dicotiledôneas, mantendo-se a seletividade às plantas cultivadas na fase inicial de crescimento (Honda \& Honda, 1990).

De modo geral, estudam-se herbicidas utilizados na cultura da soja, pois elas têm comportamentos semelhantes pelo fato de pertencerem à mesma família. No campo, a alfafa tem-se mostrado mais tolerante, porem o número de produtos químicos desenvolvidos para a soja é maior, em função da importância econômica.

Segunda Lorenzi (1994), apenas diquat, diuron, clethodim, EPTC e amônio glufosinato são registrados no Brasil para uso na cultura da alfafa. Rodrigues et al., (1995) relacionam os herbicidas recomendados para a cultura da alfafa: diuron,
Key words: Medicago sativa, green house, chemical control. EPTC, ioxynil octanoato, metribuzin, napropamide e sethoxydim. De acordo com Nuernberg et al. (19900 os herbicidas e respectivas doses ( $\mathrm{kg}$ ou $\mathrm{L} / \mathrm{ha}$ ) que podem ser usados na cultura as alfafa são: EPTC $(4,0-6,0)$, prometryne $(1,2-2,5)$, diuron $(1,5-2,5)$, diquat $(1,5-2,0)$ e trifluralin $(1,2-2,4)$.

Diante do exposto, objetivou-se avaliar, em casa de vegetação, a seletividade de diferentes herbicidas e doses, aplicados em pós-emergência das plantas de alfafa.

\section{MATERIAL E MÉTODOS}

\section{Experimento 1}

Foram realizados dois experimentos, sendo que no primeiro (Exp. 1) testaram-se vários herbicidas gramicidas e latifolicidas já recomendados para outras culturas e no segundo (Exp. 2) foram estudados apenas os herbicidas que mostraram-se mais promissores no anterior, em várias doses.

$\mathrm{O}$ experimento foi conduzido em casa de vegetação pertencente ao Departamento de Defesa Fitossanitária da Faculdade de Ciências Agrárias e Veterinárias, Câmpus de Jaboticabal/UNESP, no período de 10/03/95 a 01/06/95. O cultivar de alfafa utilizado foi o Crioula, sendo as sementes inoculadas com Rhizobium melilloti, conforme as recomendações para a cultura.

A semeadura foi realizada no dia 17/03/95, em vasos que continham aproximadamente $4 \mathrm{~kg}$ de textura argilosa, adubado com 2,5g da fórmula 2-30-10 por $\mathrm{kg} \mathrm{da}$ mesmo. Os vasos foram irrigados diariamente, de acordo com a necessidade das plantas. Após a germinação realizou-se o desbaste, deixando-se quatro plantas por vaso. Os herbicidas, bem como as doses avaliadas, estão listados na Tabela 1. Os herbicidas aplicados em 07/04/95, aos 21 dias após a semeadura (DAS), quando as plantas apresentavam aproximadamente $15 \mathrm{~cm}$ de altura e 
4 a 5 folhas trifoliadas. A aplicação foi realizada no período da manhã, com temperatura de $25^{\circ} \mathrm{C}$ e umidade relativa do ar de $70 \%$. Para a aplicação dos herbicidas, utilizou-se de um pulverizador costal, à pressão constante (proporcionada pelo $\mathrm{CO}_{2}$ comprimido) de 35 $\mathrm{lbf} / \mathrm{pol}^{2}$, munido com barra e dois bicos de jato plano ('leque") 11003, espaçados de $0,5 \mathrm{~m}$. O consumo de calda foi equivalente a 250 1/ha.

TABELA 1. Herbicidas e dosagens avaliadas, em casa de vegetação, no Experimento 1.

\begin{tabular}{llc}
\hline \multicolumn{1}{c}{ Nome comum } & \multicolumn{1}{c}{ Herbicida } & \multirow{2}{*}{ Dosagem $(\mathrm{g} / \mathrm{ha}+\%)^{*}$} \\
\hline MSMA & Daconate & 1440,0 \\
Clethodim + óleo mineral & Select + Dytrol & $96,0+0,5$ \\
Lactofen & Cobra & 144,0 \\
Fluazifop-p-butil & Fusilade & 125,0 \\
Fomesafen + óleo mineral & Flex + Energic & $250,0+0,2$ \\
Haloxyfop-methyl + óleo mineral & Verdict + Joint & $125,0+0,5$ \\
Fenoxaprop-ethyl & Furore & 120,0 \\
Chlorimuron-ethyl & Classic & 17,5 \\
Halosulfuron + óleo mineral & Sempra + Aquamix & $112,5+0,5$ \\
Nicosulfuron & Sanson & 60,0 \\
Acifluorfen & Tackle & 255,0 \\
Imazethapyr & Pivot & 100,0 \\
Bentazon+ óleo mineral & Basagran 600 + Assist & $600,0+0,5$ \\
Cyanazine + simazine & Blazina & $1500,0+1500,0$ \\
Testemunha & & - \\
\hline
\end{tabular}

$* \mathrm{~g} / \mathrm{ha}+\%=$ gramas de ingrediente ativo por equitare $+\%$ de adjuvante na calda

Foram feitas avaliações visuais de toxicidade aos 5, 12 e 21 dias após a aplicação dos herbicidas (DAA), baseando-se na escala EWRC (1964). Nesta escala a nota 1 significa ausência de fitotoxicidade e 9 a morte das plantas. Aos 21 DAA, ou seja, 42 dias após a semeadura, avaliouse a altura das plantas e realizou-se o $1^{\circ}$ corte da parte aérea, a $5 \mathrm{~cm}$ do nível do solo. As amostras foram postas a secar em estufa de circulação forçada, na temperatura de $70^{\circ} \mathrm{C}$ até o peso constante, determinando-se o peso de matéria seca. Aos 41 DAA, quando as plantas atingiram 20 dias de rebrota, realizou-se o $2^{\circ}$ corte (18/05/95), determinando-se os mesmos pesos, conforme metodologia já descrita anteriormente.

$\mathrm{O}$ delineamento experimental utilizado foi o inteiramente casualizado, com três repetições. Os dados obtidos foram submetidos à análise de variância pelo teste $\mathrm{F}$ e as médias comparadas pelo teste de Tukey, a nível de 5\% de probabilidade.
Para a análise, os dados foram transformados em $\operatorname{arcsem} \sqrt{x+0,5}$.

\section{Experimento 2}

$\mathrm{O}$ experimento foi conduzido em casa de vegetação, no período de 07/05/95 a 10/08/95, adotando-se os mesmos procedimentos do anterior, variando-se os herbicidas e suas dosagens e considerando-se os que proporcionaram os melhores resultados no Experimento 1.

A semeadura da alfafa foi realizada em 10/05/95, seguindo-se o mesmo esquema de preparação dos vasos descritos no Experimento 1. Os herbicidas avaliados e as respectivas dosagem estão listados na Tabela 2.

Os herbicidas foram aplicados em 02/06/95 923 dias após a semeadura), quando as plantas tinham 5 a 6 trifólios e aproximadamente $15 \mathrm{~cm}$ de altura. 
TABELA 2. Herbicidas e dosagens avalliadas, em casa de vegetação, no Experimento 2.

\begin{tabular}{llc}
\hline & Herbicida & Dosagem $(\mathrm{g} / \mathrm{ha}+\%)^{*}$ \\
\hline Nome comum & Nome comercial & 960,0 \\
\hline MSMA & Daconate & 1440,0 \\
MSMA & Daconate & 1920,0 \\
MSMA & Daconate & 12,5 \\
Chlorimuron-ethyl & Classic & 15,0 \\
Chlorimuron-ethyl & Classic & 17,5 \\
Chlorimuron-ethyl & Classic & 50,0 \\
Imazethapyr & Pivot & 75,0 \\
Imazethapyr & Pivot & 100,0 \\
Imazethapyr & Pivot & 600,0 \\
Bentazon & Basagran 600 & 900,0 \\
Bentazon & Basagran 600 & 1200,0 \\
Bentazon & Basagran 600 & $900,0+0,5$ \\
Bentazon + óleo mineral & Basagran 600 + Assist & 96,0 \\
Clethodim & Select & $96,0+0,5$ \\
Clethodim + óleo minral & Select + Dytrol & $720,0+960,0$ \\
Bentazon + MSMA & Basagran 600 + Daconate & \\
Testemunha & & - \\
\hline
\end{tabular}

$* \mathrm{~g} / \mathrm{ha}+\%=$ gramas de ingrediente ativo por equitare $+\%$ de adjuvante na calda

A aplicação foi feita com temperatura de $25^{\circ} \mathrm{C}$ e umidade relativa do ar de $60 \%$. Utilizou-se do mesmo pulverizador costal e as características da aplicação foram idênticas as do Experimento 1.

Foram feitas avaliações visuais dos sintomas de intoxicação nas plantas de alfafa aos 14 dias após a aplicação dos herbicidas (DAA), baseando-se escala de notas EWRC (1964). No dia 03/07/95, aos 54 dias após a semeadura, avaliou-se a altura das plantas e a produção de matéria seca, após o $1^{\circ}$ corte (03/07/95), feito a $5 \mathrm{~cm}$ do nível do solo, conforme metodologia já descrita anteriormente. No dia 01/08/95 foi realizado o $2^{\circ}$ corte, com 28 dias de rebrota, adotando-se os mesmos procedimentos descritos anteriormente. $\mathrm{O}$ delineamento experimental utilizado foi o inteiramente casualizado, com três repetições. Os dados obtidos, após transformados, foram submetidos a análise de variância pelo teste $\mathrm{F}$ e as médias comparadas pelo Teste de Tukey, a nível de $5 \%$ de probabilidade.

\section{RESULTADOS E DISCUSSÃO}

Na Tabela 3 são apresentados os dados referentes à produção de matéria seca altura de plantas e notas de fitoxicidade para a alfafa tratada com diferentes herbicidas, em casa de vegetação (Experimentação1).

A análise dos dados da Tabela 3 mostra que as plantas tratadas com herbicidas graminicidas, tais como fluazifop-p-butil, fenoxaprop-ethyl e haloxyfop-methyl, foram as que receberam as menores notas de fitointoxicação, sem interferência também, na altura das plantas. O mesmo resultado não foi observado para as plantas que receberam clethodim, pois embora sendo um graminicida específico, foi fitotóxico para a alfafa. Os produtos químicos que apresentaram fitotoxicidade intermediária foram MSMA, chlorimuron-ethyl e imazethapyr. Os herbicidas cyanazine + simazine, lactofen, fomesafen, halosulfuron, acifluorfen, nicosulfuron e bentazon 
+ óleo mineral apresentaram notas de maiores fitointoxicaçoes e reduções na altura das fitotoxicidade superiores a 6 (seis), com as plantas.

TABELA 3. Produção de matéria seca, altura e notas de fitotoxicidade, em plantas de alfafa tratadas com diferentes herbicidas, na casa de vegetação (Experimento 1).

\begin{tabular}{|c|c|c|c|c|c|c|c|}
\hline \multicolumn{2}{|l|}{ Herbicidas } & \multicolumn{2}{|c|}{$\begin{array}{c}\text { Produção de matéria } \\
\text { seca (g/vaso) }\end{array}$} & \multirow{2}{*}{$\begin{array}{l}\text { Altura }(\mathrm{cm}) \\
21 \mathrm{DAA}^{* * *}\end{array}$} & \multicolumn{3}{|c|}{$\begin{array}{c}\text { Notas (EWRC) } \\
\text { DAA }\end{array}$} \\
\hline Nome comum & $\begin{array}{c}\text { Dosagem } \\
(\mathrm{g} / \mathrm{ha}+\%)^{*}\end{array}$ & $\begin{array}{c}1^{\circ} \text { Corte } \\
\left(42 \mathrm{DAS}^{* *}\right)\end{array}$ & $\begin{array}{l}2^{\circ} \text { Corte } \\
\text { (62 DAS) }\end{array}$ & & 5 & 12 & 21 \\
\hline MSMA & 1440,0 & $3,2 \mathrm{BC}$ & $4,8 \mathrm{BC}$ & 25,2 ABCD & 3,3 & 2,7 & 3,3 \\
\hline Clethodim+óleo mineral & $96,0+0,5$ & $1,4 \mathrm{DEF}$ & $3,4 \mathrm{C}$ & $16,9 \mathrm{CDEF}$ & 7,7 & 6,0 & 6,0 \\
\hline Lactofen & 144,0 & $0,0 \quad \mathrm{G}$ & $0,0 \mathrm{E}$ & $1,7 \mathrm{GH}$ & 8,7 & 9,0 & 9,0 \\
\hline Fluazifop-buthyl & 125,0 & $4,0 \quad \mathrm{~B}$ & $5,0 \quad \mathrm{~B}$ & $31,0 \mathrm{ABC}$ & 2,0 & 1,0 & 1,0 \\
\hline Fomesafen+óleo mineral & $250,0+0,2$ & $0,0 \quad \mathrm{G}$ & $0,2 \mathrm{E}$ & $2,7 \mathrm{GH}$ & 9,0 & 9,0 & 9,0 \\
\hline Fenoxaprop-ethyl & 120,0 & 3,9 B & $4,7 \quad \mathrm{~B}$ & $34,5 \quad \mathrm{AB}$ & 1,7 & 1,0 & 1,0 \\
\hline Haloxyfop-methyl + óleo mineral & $125,0+0,5$ & $4,1 \quad \mathrm{~B}$ & $4,0 \mathrm{BC}$ & $36,5 \mathrm{~A}$ & 1,3 & 1,0 & 1,0 \\
\hline Chlorimuron-ethyl & 17,5 & 2,1 CDE & $3,5 \mathrm{C}$ & $19,5 \mathrm{BCDEF}$ & 5,3 & 4,0 & 4,7 \\
\hline Halosulfuron + óleo mineral & $112,5+0,5$ & $0,0 \quad \mathrm{G}$ & $0,1 \mathrm{E}$ & 5,0 FGH & 6,0 & 7,0 & 8,7 \\
\hline Cyanazine + simazine & $1,5+1,5$ & $0,0 \quad \mathrm{G}$ & $0,0 \mathrm{E}$ & $0,0 \quad \mathrm{H}$ & 6,0 & 9,0 & 9,0 \\
\hline Bentazon + óleo mineral & $600,0+0,5$ & $0,0 \quad \mathrm{G}$ & $0,2 \mathrm{E}$ & 7,7 EFGH & 3,3 & 8,0 & 8,7 \\
\hline Nicosulfuron & 60,0 & $0,2 \quad \mathrm{FG}$ & $0,0 \mathrm{E}$ & 12,7 DEFGH & 6,7 & 7,0 & 8,3 \\
\hline Acifluorfen & 255,0 & $1,0 \quad \mathrm{EFG}$ & $2,1 \quad \mathrm{D}$ & 12,1 DEFGH & 7,7 & 7,0 & 7,0 \\
\hline Imazethapyr & 100,0 & $2,4 \quad \mathrm{CD}$ & $4,2 \mathrm{BC}$ & 22,3 ABCDE & 3,3 & 4,0 & 4,3 \\
\hline Testemunha & & $5,4 \quad \mathrm{~A}$ & $6,2 \quad \mathrm{~A}$ & $37,2 \mathrm{~A}$ & 1,0 & 1,0 & 1,0 \\
\hline $\mathrm{Cv}(\%)$ & & 23,3 & 15,6 & 29,0 & & & \\
\hline
\end{tabular}

Médias seguidas de mesma letras nas colunas não diferem $(\mathrm{P}>0,05)$, pelo teste de Tukey

$* \mathrm{~g} / \mathrm{ha}+\%=$ gramas de ingrediente ativo por equitare $+\%$ de adjuvante na calda

**DAS = dias após a semeadura

***DAA $=$ dias após a aplicação

Quanto à produção de matéria seca (PMS), observou-se que todos os herbicidas proporcionaram reduções significativas na PMS da parte aérea das plantas, no primeiro e segundo cortes. As maiores reduções na PMS, em relação à testemunha, foram obervadas com o uso de lactofen, fomesafen, halosulfuron, cyanazine + simazine, bentazon, nicosulfuron, aciflurfen e clethodim. Acifluorfen proporcionou $66 \%$ de redução na PMS no segundo corte, enquanto que cyanazine + simazine, halosulfuron, lactofen, fomesafen, nicosulfuron e bentazon proporcionaram reduções na PMS acima de 96\%, o que representou alta fitoxicidade para a cultura. Dower Neto et al. (1988), avaliando bentazon a $720 \mathrm{~g} / \mathrm{ha}$, fomesafen a $250 \mathrm{~g} / \mathrm{ha}$, bentazon a 480 $\mathrm{g} / \mathrm{ha}+$ fomesafen a $200 \mathrm{~g} / \mathrm{ha}$, bentazon a $480 \mathrm{~g} / \mathrm{ha}$ + fomesafen a $250 \mathrm{~g} / \mathrm{ha}$, sethoxydim a $276 \mathrm{~g} / \mathrm{ha} \mathrm{+}$ fomesafen a $250 \mathrm{~g} / \mathrm{ha}$, sethoxydim a $276 \mathrm{~g} / \mathrm{ha}+$ lactofen a $72 \mathrm{~g} / \mathrm{ha}$, lactofen a $48 \mathrm{~g} / \mathrm{ha}+$ acifluorfen a $320 \mathrm{~g} / \mathrm{ha}$ e bentazon a $96 \mathrm{~g} / \mathrm{ha}+$ paraquat a 64 $\mathrm{g} / \mathrm{ha}$, também verificaram que todos os herbicidas causaram redução na PMS da alfafa, comparados ao controle.

No presente estudo os herbicidas mais seletivos para alfafa foram o haloxyfopmethyl, o fluazifop-p-butil e o fenoxaprop-ethyl com resultados semelhantes aos obtidos por Ott et al. (984), que estudaram o controle de Triticum aestivum associado à cultura com o segundo na dose de $120 \mathrm{~g} / \mathrm{ha}$. Esses mesmos autores também testaram o sethoxydim a $180 \mathrm{~g} / \mathrm{ha}$ e não obtiveram redução na produtividade quando usaram até 3,2 $\mathrm{kg} / \mathrm{ha}$ destes berbicidas. Hartwing (1986) conseguiu também controle adequado de Setaria faberii com fluazifop-p-buthyl e fenoxaprop, sem prejuízos para a cultura da alfafa. Beardmore \& Linscott (1988) e Moyer \& Schaalje (1993) 
mostraram que fluazifop-p-buthul e haloxyfopmethyl são seletivos à alafafa. Honda \& Honda (1990) afimaram que fluazifop-p-buthyl, haloxyfop-methyl e sethoxydim não causaram danos às brotações das plantas de alfafa.

Os herbicidas com efeitos fitotóxicos intermediários foram MSMA, imazethapyr e chlorimuron-ethyl, que proporcionaram reduções ma PMS de 40,4; 55,0 e 60,2\% no primeiro corte, sendo que no segundo elas foram de 34,$3 ; 44,1$; e $32,5 \%$, respectivamente, caracterizando-se diminuição dos efeitos negativos. Esses resultados são concordantes com ao trabalhos que têm demonstrado ser alfafa, no início de seu desenvolvimento, muito sensível à ação dos herbicidas, principalmente aos produtos com ação sobre latifoliadas. Redução de $28 \%$ na produção de matéria seca no primeiro corte da alfafa foi observado por Aranha \& Miquelão (1993), quando aplicaram $15 \mathrm{~g} / \mathrm{ha}$ de chlorimuron-ethyl, sendo esta dose menor que a usada neste experimento, que foi de $17,5 \mathrm{~g} / \mathrm{ha}$. O uso de imazethapyr na dosagem de 105,36 g/ha, aplicado isoladamente, causou redução no crescimento da alfafa e, quando aplicado em mistura de tanque com bentazon, provocou clorose e diminuição no desenvolvimento das plantas (Zamora \& Alby, 1991).

Em relação ao bentazon, embora a literatura mencione que a adição de óleo mineral aumente a sua fitoxicidade para as plantas de alfafa (Harvey, 1991), não era esprado fitoxicidade tão elevada, com morte das plantas. Esse resultado, como também a fitoxicidade observada para o clethodim + óleo mineral, sugere que pode Ter ocorrido algum tipo de contaminação no momento da aplicação, embora deva-se considerar que as altas temperaturas (máximas de até $38^{\circ} \mathrm{C}$ ) observadas na casa de vegetação, no período da tarde, também possam ter influenciado na ação de tais herbicidas e consequentemente, na tolerância das plantas. Em função dos resultados obtidos desenvolveu-se outro experimento em casa de vegetação, para identificar-se as dosagens adequadas de MSMA, chlorimuron-ethyl e imazethaphyr que apresentaram potencial de uso na alfafa. Testaram-se, também, o clethodim e o bentazon, com e sem óleo mineral, para comprovar-se ou não os efeitos fitotóxicos ocorridos no Experimento 1.

Os resultados obtidos no Experimento 2 são apresentados na Tabela 4 e. após a análise dos mesmos, observa-se que a produção de matéria seca foi reduzida pelos diferentes herbicidas. Pode-se verificar que, no primeiro corte, a aplicação do bentazon a $1200 \mathrm{~g} / \mathrm{ha}$ causou maior redução na $\mathrm{PMS}) \mathrm{P}<0,05)$ e recebeu a Segunda maior nota de fitointoxicação (nota 5). Os outros herbicidas que também reduziram significativamente, em relação à testemunha, a PMS do $1^{\circ}$ Corte, foram o bentazon + MSMA, bentazon isolado a 600 e $900 \mathrm{~g} / \mathrm{ha}$ e com óleo mineral, imazethapyr (nas duas maiores doses) e chlorimuron-ethyl em todas as dosagens.

Por outro lado, no segundo corte observou-se que, apesar das produções variarem significativamente, muitas estão com valores próximos, mostrando recuperação das plantas aos afeitos dos herbicidas, pois as produções de matéria seca aumentaram, do $1^{\circ}$ para $2^{\circ}$ corte, em todos os tratamentos. Para o chlorimuron-ethyl, mantiveram-se as reduções independentemente das doses, com comportamento semelhante ao do primeiro corte. Quanto ao imazethapyr, observouse que apenas a dosagem maior, de $100 \mathrm{~g} / \mathrm{ha}$, reduziu a produção de matéria seca, caracterizando-se aumento na tolerância das plantas melhor estabelecidas.

É importante ressaltar que o tratamento com MSMA, em todas as doses, não mais afetaram a produção de matéria seca no segundo corte, caracterizando-se incremento na tolerância e permitindo vislumbrar-se o potencial de uso do herbicida nesta cultura.

Em relação às notas de fitoxicidade, que foram atribuídas aos 14 dias após a aplicação, foram registrados valores mais elevados com a aplicação de chlorimuron-ethyl e bentazon, isolado e na maior dosagem (1200 g/ha) ou com óleo mineral. Em relação a altura das plantas, coerentemente observou-se redução em reposta à aplicação do herbicida chlorimuron-ethyl nas duas 
maiores doses $(15,0$ e $17,5 \mathrm{~g} / \mathrm{ha})$. para o clethodim, isolado ou com óleo mineral, a altura foi estatisticamente a mesma da testemunha, mostrando que ele e o bentazon, mesmo com o adjuvante, não são tão fitotóxicos, como ocorreu no Experimento 1.

TABELA 4. Produção de matéria seca, altura e notas de fitotoxicidade, em plantas de alfafa tratadas com diferentes herbicidas, na casa de vegetação (Experimento 2).

\begin{tabular}{|c|c|c|c|c|c|}
\hline \multicolumn{2}{|c|}{ Herbicidas } & \multicolumn{2}{|c|}{$\begin{array}{c}\text { Produção de matéria seca } \\
\text { (g/vaso) }\end{array}$} & Altura $(\mathrm{cm})$ & \multirow{2}{*}{$\begin{array}{l}\text { Notas (EWRC) } \\
14 \text { DAA }\end{array}$} \\
\hline Nome comum & $\begin{array}{l}\text { Dosagem } \\
(\mathrm{g} / \mathrm{ha}+\%)\end{array}$ & $\begin{array}{c}1^{\circ} \text { Corte } \\
(54 \text { DAS } * *)\end{array}$ & $\begin{array}{l}2{ }^{\circ} \text { Corte } \\
(83 \text { DAS })\end{array}$ & $28 \mathrm{DAA}^{* * *}$ & \\
\hline MSMA & 960,0 & $6,6 \mathrm{AB}$ & $8,2 \mathrm{ABC}$ & $36,4 \quad \mathrm{AB}$ & 2,0 \\
\hline MSMA & 1440,0 & $4,3 \quad \mathrm{BCD}$ & 7,4 ABCDE & $31,2 \mathrm{AB}$ & 3,0 \\
\hline MSMA & 1920,0 & $5,4 \quad \mathrm{ABCD}$ & 8,9 A & $28,6 \mathrm{AB}$ & 4,0 \\
\hline Chlorimuron-ethyl & 12,5 & $3,6 \mathrm{CD}$ & $4,5 \mathrm{E}$ & $30,1 \quad \mathrm{AB}$ & 5,0 \\
\hline Chlorimuron-ethyl & 15,0 & $3,8 \mathrm{CD}$ & $5,1 \quad \mathrm{DE}$ & $25,6 \quad \mathrm{~B}$ & 5,0 \\
\hline Chlorimuron-ethyl & 17,5 & $3,8 \quad \mathrm{CD}$ & $5,2 \mathrm{CDE}$ & $26,5 \mathrm{~B}$ & 6,0 \\
\hline Imazethapyr & 50,0 & $5,5 \mathrm{ABCD}$ & $8,0 \quad \mathrm{ABCD}$ & $33,3 \quad \mathrm{AB}$ & 1,0 \\
\hline Imazethapyr & 75,0 & $4,6 \mathrm{BCD}$ & 6,6 ABCDE & $35,9 \quad \mathrm{AB}$ & 2,0 \\
\hline Imazethapyr & 100,0 & $4,1 \quad \mathrm{BCD}$ & $5,7 \mathrm{CDE}$ & $32,4 \quad \mathrm{AB}$ & 3,0 \\
\hline Bentazon & 600,0 & $4,5 \mathrm{BCD}$ & 7,6 ABCD & $31,5 \mathrm{AB}$ & 3,0 \\
\hline Bentazon & 900,0 & $3,7 \quad \mathrm{CD}$ & 6,1 ABCDE & $26,0 \quad \mathrm{~B}$ & 4,0 \\
\hline Bentazon & 1200,0 & $3,4 \mathrm{D}$ & 5,8 BCDE & $29,7 \quad \mathrm{AB}$ & 5,0 \\
\hline Bentazon + óleo mineral & $900,0+0,5$ & $4,2 \mathrm{BCD}$ & 6,9 ABCDE & 31,2 & 5,0 \\
\hline Clethodim & 96,0 & 5,9 ABCD & $8,1 \quad \mathrm{ABC}$ & $36,7 \quad \mathrm{AB}$ & 1,0 \\
\hline Clethodim + óleo mineral & $96,0+0,5$ & $6,1 \quad \mathrm{ABC}$ & 7,4 ABCDE & $41,0 \quad \mathrm{~A}$ & 1,0 \\
\hline Bentazon+MSMA & $720,0+960,0$ & $3,5 \mathrm{CD}$ & 6,1 ABCDE & $29,7 \quad \mathrm{AB}$ & 4,0 \\
\hline Testemunha & - & $7,8 \mathrm{~A}$ & $8,8 \quad \mathrm{AB}$ & $39,8 \mathrm{~A}$ & 1,0 \\
\hline C.V. $(\%)$ & & 18,6 & 14,4 & & \\
\hline
\end{tabular}

Médias seguidas de mesmas letras nas colunas não diferem ( $\mathrm{p}>0,05)$ pelo teste de Tukey

$* \mathrm{~g} / \mathrm{ha}+\%=$ gramas de ingrediente ativo por equitare $+\%$ do adjuvante na calda

$* * \mathrm{DAS}=$ dias após a semeadura

***DAA $=$ dias após a aplicação

\section{LITERATURA CITADA}

ARANHA, M.T.M., MIQUELÃO, M.E. Controle de plantas daninhas na cultura da alfafa. In: CONGRESSO BRASILEIRO DE HERBICIDAS E PLANTAS DANINHAS, 19, 1993. Londrina. Resumos... Londrina:SBHED, 1993. p.237-238.

BEARDMORE, R.A., LINSCOTT, T.L. Postemergence herbicide supression of wheat, triticum aestivum, growing with alfafa, Medicago sativa L. Weed Sci., v.36, n., p.636-641, 1988.
DAWSON, J.H., RINCKER, C.M. Weeds in new seedlings of alfalfa (Medicago sativa) for seed production: competition and control. Weed Sci., v.30, n., p.20-25, 1982.

DURIGAN, J.C. Matocompetição e comportamento de baixas doses de herbicidas, na cultura da soja [Glycine max (L.) Merril]. ESALQ/USP, Piracicaba, 1993, 163p. (Teses de Doutorado).

EWRC (European Weed Research Council). Report of the $3^{\text {rd }}$ and 4 th meetings of EWRC - Comitee of Methods in Weed Research. Weed Res., v.4, n. 1., p.88, 1964. 
HARTWING, N.L. Safety offenoxaprop and fluazifop on alfafa-cool season grass seedlings. In ANNUAL MEETING OF THE NORTHEASTERN WEED SCIENCE SOCIETY, 40, 1986, Proceedings..., p.5556.

HARVEY, R.G. Bentazon for annual control in newly seeded alfafa (Medicago sativa). Weed Technol., v.5, n.1, p.154-158, 1991.

HONDA, C.S., HANDA, A.M. Cultura da alfafa Marília: Artes Gráficas, 1990. 245p.

LONREZI, H. Manual de identificação e controle de plantas daninhas: plantio direto e convencional. 4 ed., Nova Odessa; SP, 1994. 175p.
MOYER, J.R. \& SCHAALJE, G.B. Quackgrass (Elytrigia repens) interference and control in seed alfafa (Medicargo sativa). Weed Technol., v.7, n., p.58-64, 1993.

OTT,P., DAWSON, J.H., APPLEBY, A.P. Control selectivo de trigo "gaucho"(Triticum aestivum L.) en alfafa (Medicago sativa L.) a la implantación, com fluazifop-p-butil y sethoxydim. In: CONGRESSO BRASILEIRO DE HERBICIDA E PLANTAS DANINHAS, 15, 1984, Belo Horizonte. Resumos..., Londrina: SBHED, 1984. p.155-156.

ZAMORA, D., ALBY, T. Weed control in seedling alfafa with imazethapyr. In: Western Society of Weed Science, 44, 1991, Seatle. Proceeding, p.97-98. 